\title{
Fenilcetonúria Materna: Relato de Caso
}

\author{
Maternal Phenylketonuria: A Case Report
}

\begin{abstract}
Ernesto Antonio Figueiró-Filho, Alessandro Henrique Antunes Lopes, Flávio Renato de Almeida Senefonte, Virgilio Gonçalves de Souza Júnior, Carlos Augusto Botelho, Geraldo Duarte
\end{abstract}

\section{RESUM0}

A fenilcetonúria materna é uma aminoacidopatia caracterizada por níveis elevados de fenilalanina plasmática na gestante, o que pode provocar anormalidades no desenvolvimento do feto, condição que se denomina síndrome de fenilcetonúria materna. Deve ser diagnosticada laboratorialmente, uma vez que as manifestações clínicas são inespecíficas. Relatamos um caso de paciente secundigesta, com antecedente pessoal de retardo do desenvolvimento cognitivo, sem antecedentes patológicos obstétricos, com diagnóstico laboratorial de hiperfenilalaninemia na atual gestação, sendo tratada com dieta específica. O recémnato, nascido a termo, não apresentou alterações físicas ou defeitos congênitos confirmados. A gestação anterior, na qual não houve diagnóstico e controle da fenilcetonúria, resultou em criança com séria deficiência psicomotora confirmada, além de microcefalia e distúrbios auditivos e da fala. Com o conhecimento dos efeitos da hiperfenilalaninemia materna sobre o feto, tornam-se essenciais o diagnóstico e a instituição precoce do tratamento durante a gravidez em pacientes com suspeita clínica de fenilcetonúria. No caso aqui descrito, houve beneficios materno-fetais do tratamento dietoterápico oferecido, reforçando a importância da identificação de mulheres fenilcetonúricas em idade reprodutiva.

PALAVRAS-CHAVE: Fenilcetonúria. Erros inatos do metabolismo. Hiperfenilalaninemia. Gestação de alto risco.

\section{Introdução}

As hiperfenilalaninemias (HPA) são erros inatos do metabolismo, de herança autossômica recessiva, cujo distúrbio primário se localiza na conversão do aminoácido fenilalanina em tirosina por deficiência da enzima hepática fenilalaninahidroxilase (PAH). Em conseqüência disto, ocorre aumento da concentração de fenilalanina e de seus subprodutos no sangue e na urina (fenilpiruvato, fenilacetato, fenilactato e fenilacetilglutamina),

Ambulatório de Gestação de Alto-Risco do Centro de Atendimento Multidisciplinar de Saúde (CAMS-APAE) Campo Grande - MS. Universidade Federal de Mato Grosso do Sul.

Correspondência:

Ernesto Antonio Figueiró-Filho

Rua Amazonas, 649 - Bairro São Francisco

79010-060 - Campo Grande - MS

Fone: (67) 3029-2862 - (67) 325-7829

e-mail: eafigueiro@uol.com.br com formação reduzida de tirosina ${ }^{1}$. Graves danos cerebrais são acarretados pela elevação da concentração plasmática de fenilalanina no organismo, tais como a inibição competitiva do transporte de outros aminoácidos necessários para a síntese de proteínas, formação ou estabilização debilitadas de polirribossomos, síntese reduzida e degradação aumentada de mielina, bem como formação inadequada de norepinefrina e serotonina ${ }^{1}$.

Classificam-se as HPA $\mathrm{em}^{1}$ : fenilcetonúria (PKU) clássica: atividade da $\mathrm{PAH}$ inferior a $1 \% \mathrm{e}$ fenilalanina plasmática superior a $20 \mathrm{mg} / \mathrm{dL}$ $(\geq 1200 \mu \mathrm{mol} / \mathrm{L})$; PKU leve: atividade da PAH de 1 a $3 \%$ e fenilalanina plasmática entre $10 \mathrm{e}$ $20 \mathrm{mg} / \mathrm{dL}$ (entre 600 e $1200 \mu \mathrm{mol} / \mathrm{L}$ ); hiperfenilalaninemia permanente ou transitória: atividade da PAH superior a $3 \%$ e fenilalanina plasmática entre 4 e $10 \mathrm{mg} / \mathrm{dL}$ (entre 240 e $600 \mu \mathrm{mol} / \mathrm{L}$ ).

A PKU ocorre em todos os grupos étnicos. Devido à grande variabilidade genética, a incidência em recém-nascidos (RN) pode variar de 1:2.600 
a 1:26.000, obtendo-se em média o índice de $1: 10.000 /$ nascidos vivos $^{2}$. A estimativa nacional, baseada em estudo realizado no Rio Grande do Sul, indica prevalência de um caso para cada 12.000 nascidos vivos ${ }^{3}$.

Em virtude do diagnóstico precoce e da dieta com restrição de fenilalanina durante a infância, muitas mulheres atingem a idade fértil. Sendo assim, torna-se possivel a identificação de gestantes portadoras de PKU, condição grave que, quando não diagnosticada e tratada, leva à síndrome de PKU materna. Tal síndrome manifesta-se no feto por restrição do crescimento intra-uterino (RCIU), microcefalia, retardo mental e malformações cardíacas congênitas ${ }^{4}$. Nas gestantes portadoras de PKU, podem-se encontrar graus variados de deficiência do desenvolvimento cognitivo, associando-se ou não a convulsões e hiperatividade ${ }^{1}$. Em decorrência dessas possiveis alterações maternas e fetais, torna-se de vital importância a detecção da PKU em gestantes.

\section{Relato de Caso}

E.G.S., 22 anos, pele, olhos e cabelos claros, Gesta 2, Para 1 (parto cesárea), tempo de amenorréia de 10 semanas e 2 dias, procedente da zona rural de Rio Brilhante - MS, casada, alfabetizada (ensino fundamental completo), sem antecedentes pessoais patológicos (inclusive obstétricos). Foi admitida para consulta pré-natal no dia 27 de janeiro de 2003 em sua localidade de origem. Foram solicitados os exames de rotina prénatal do Programa Estadual de Proteção à Gestante de Mato Grosso do Sul, entre os quais se inclui a dosagem de fenilalanina no sangue materno. Foi constatada concentração de fenilalanina plasmática igual a $24,3 \mathrm{mg} / \mathrm{dL}$ (normal $<4 \mathrm{mg} / \mathrm{dL}$ ).

Foi encaminhada ao ambulatório de gestação de alto-risco do Centro de Atendimento Multidisciplinar de Saúde (CAMS-APAE/UFMS) em Campo Grande, sendo admitida em 14 de fevereiro de 2003. No momento da consulta encontravase com 12 semanas e 6 dias de gestação e referia parto cesáreo anterior. O RN, do sexo feminino, nascido prematuramente há seis anos, pesou $1.400 \mathrm{~g}$, apresentou microcefalia e evoluiu com retardo do desenvolvimento psicomotor.

Foram realizados, durante o pré-natal atual, os seguintes exames maternos: hemograma $(\mathrm{Hb}$ : 12,2 g/dL; Ht: 35,2 mL/dL), VDRL (negativo), urina I (sem alterações), exame parasitológico de fezes (sem alterações), glicemia $(78 \mathrm{mg} / \mathrm{dL})$, citologia oncótica (sem alterações), sorologia para HIV (ne- gativa), toxoplasmose (IgM negativo; IgG positivo), rubéola (IgM negativo; IgG positivo), pesquisa do antígeno capsular do vírus $\mathrm{B}$ da hepatite/HbsAg (negativo), anticorpos contra o virus $\mathrm{C}$ da hepatite/ anti-HCV (negativo), sorologia anti-IgM para o vírus herpes simplex 1-2 (negativo) e doença de Chagas (negativo), pesquisa de anticorpos IgM para citomegalovírus (negativo) e GTT75 g (72/100). Com 13 semanas foi realizada ultra-sonografia indicando translucência nucal de $1,0 \mathrm{~mm}$.

Durante o período pré-natal, instituiu-se dieta específica para portadores de PKU, com restrição de fenilalanina. Esta dieta $^{5}$, orientada por nutricionista do grupo, consistia da retirada de proteínas de alto valor biológico da dieta, sendo proscritos os grãos (feijão, ervilha, lentilha, soja, grão-de-bico, trigo e gérmen de trigo) e as proteínas de origem animal (leite, carne e ovos). Em substituição às proteínas retiradas da dieta foi fornecida suplementação com hidrolisado protéico (contém todos os aminoácidos essenciais, sendo isento de fenilalanina e acrescido de tirosina). Os carboidratos e lipídios de origem vegetal podem ser consumidos livremente.

Foram realizadas mais 12 consultas de prénatal paralelamente ao Ambulatório de Nutrição, sendo acompanhados periodicamente os níveis de fenilalanina plasmática materna, cujos valores encontram-se na Figura 1. Foram ainda realizados mais três exames ecográficos, demonstrando desenvolvimento fetal adequado. No dia 6 de agosto de 2003 foi realizada operação cesariana, estando a paciente com 37 semanas e 5 dias de gestação, devido à apresentação pélvica e início espontâneo de trabalho de parto com ruptura da bolsa das águas. O RN, do sexo masculino, apresentou Apgar 6 e 9 no primeiro e quinto minutos, respectivamente, pesou 2.810 g e mediu $45 \mathrm{~cm}$. Nove dias após o parto, foi coletado sangue em papel filtro para triagem neonatal do RN, não sendo constatada PKU (fenilalanina plasmática de $0,73 \mathrm{mg}$ / dL). Também não foram encontradas malformações congênitas no RN. A criança, atualmente com 1 ano de idade, está sendo acompanhada pelo serviço de pediatria do CAMS-APAE/UFMS, não apresentando alterações cardíacas e neurológicas, tendo desenvolvimento psicomotor adequado para a faixa etária até o presente momento.

\section{Discussão}

Foram extensamente documentados casos de fetos vivos com RCIU, microcefalia, malformações cardíacas congênitas e retardo mental nos 
primeiros anos de vida, cujas mães eram portadoras de PKU não tratada ${ }^{6-8}$. A fenilalanina materna em excesso atravessa a barreira placentária, atingindo níveis plasmáticos fetais de 1,2 a 1,9 vezes mais elevados que no sangue materno ${ }^{9}$. Como o sistema hepático fetal tem dificuldades em metabolizar a fenilalanina, ocorre hiperfenilalaninemia, a qual possui ação lesiva sobre o feto, sobretudo sobre o cérebro, onde são demonstradas alterações na mielinização, na síntese protéica cerebral e na produção de neurotransmissores ${ }^{10}$. Resulta, então, o desenvolvimento da síndrome de PKU materna no feto.

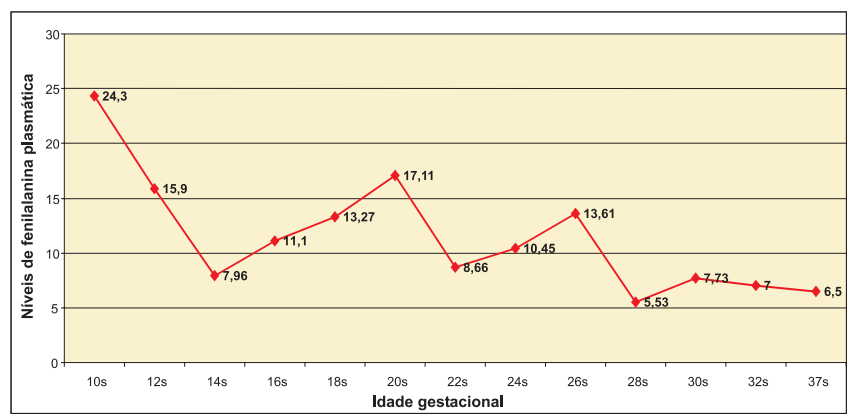

Figura 1 - Níveis de fenilalanina plasmática materna durante a gravidez.

Segundo Lenke e Levy ${ }^{10}, 92 \%$ dos filhos de gestantes com PKU não tratada apresentaram retardo mental, 73\% demonstraram microcefalia, $12 \%$ malformações cardíacas congênitas e $40 \%$ baixo peso ao nascer $(<2.500 \mathrm{~g})$. A microcefalia geralmente se acompanha de anormalidades faciais caracterizadas por face arredondada, fissuras palpebrais amplas, glabela proeminente, hipertelorismo ocular, epicanto, palato em ogiva e estrabismo ${ }^{11}$. Em 24\% das gestações ocorrem abortos espontâneos ${ }^{10}$ e em 40 a $56 \%$ dos RN de mães fenilcetonúricas pode-se observar RCIU ${ }^{12}$. O retardo mental é relatado em cerca de $21 \%$ dos RN de mães com PKU transitória, ocorrendo em mais de $92 \%$ dos RN de mães com PKU clássica ${ }^{11,13}$. Nota-se concordância do caso aqui descrito com os dados da literatura, uma vez que a gestação prévia, na qual não houve tratamento da PKU clássica materna, resultou em criança com baixo peso ao nascer, microcefalia e retardo mental. Tais alterações não foram encontradas no RN atual, em cuja gestação houve tratamento dietoterápico da PKU clássica materna.

Apesar de não haver malformações congênitas na prole da paciente aqui apresentada, existem claras evidências de que a PKU materna clássica não controlada pode exercer efeito teratogênico sobre o feto ${ }^{14}$. Dentre as malformações congênitas descritas, as mais comuns são as cardiacas, que ocorrem em 15\% dos casos, sendo a tetralogia de Fallot, coarctação da aorta, persistência do canal arterioso, defeito do septo atrial (persistência do forame oval) e hipoplasia do ventrículo esquerdo as alterações mais prevalentes $^{9,11,15}$. Segundo Michals-Malaton et al. ${ }^{7}$, a partir do Maternal Phenylketonuria Collaborative Study (MPKUCS), foi demonstrada proporção de $7,5 \%$ de defeitos cardíacos congênitos (DCC) em mulheres com PKU, sendo a incidência na população geral de $0,8 \% \%^{8,11}$. Os fetos que apresentaram DCC estavam expostos a níveis de fenilalanina acima de $10 \mathrm{mg} / \mathrm{dL}$ durante as oito primeiras semanas de gestação, sendo que em mulheres com níveis de fenilalanina abaixo de 10 $\mathrm{mg} / \mathrm{dL}$, não foram encontradas crianças com $\mathrm{DCC}^{7}$. Outras malformações associadas à PKU clássica são anomalias da coluna vertebral (cervical e sacra), fissura labial e palatina, atresia de esôfago, microftalmia e hipoplasia do corpo caloso ${ }^{16,17}$.

É importante ressaltar que na HPA branda ou persistente a morbidade fetal é menor que nos casos de mães portadoras de PKU clássica sem tratamento, e não há relatos de doenças cardíacas congênitas com incidência superior à população normal ${ }^{16}$.

Para o diagnóstico de PKU na gestação conciliam-se achados clínicos, que levarão à suspeita da doença, com o exame laboratorial confirmatório. Dentre os achados clínicos na fase adulta, pode-se encontrar retardo mental, convulsões, microcefalia, hipopigmentação e odor rançoso na pele, olhos e cabelos, e alta suscetibilidade ao eczema e outros problemas dermatológicos ${ }^{1,18}$. Além disso há história de hiperatividade, irritabilidade, atraso do desenvolvimento, distúrbios comportamentais e convulsões na infância ${ }^{18}$. Deve-se suspeitar de PKU durante a gravidez, diante de paciente com nível intelectual baixo, antecedente de filhos com retardo mental, microcefalia ou cardiopatia ${ }^{6}$, como observado no caso relatado. Para confirmação diagnóstica, deve-se determinar a concentração de fenilalanina plasmática.

As evidências demonstram que a PKU materna é fator teratogênico que pode ser prevenido ou modificado pelo tratamento dietoterápico materno $^{16,19}$. O tratamento consiste em restringir a fenilalanina da dieta, na qual alimentos de origem animal são pouco utilizados, resultando em baixa ingestão de proteínas de alto valor biológi$\mathrm{co}^{14}$. Para este fim procura-se substituir os alimentos de origem animal por verduras e frutas. Para haver adequado aporte de proteínas, suplementa-se a dieta com misturas de 
aminoácidos livres, pobres em fenilalanina mas que podem ser acrescidos de carboidratos, gorduras, minerais e vitaminas, ou como alternativa os hidrolisados protéicos isentos ou com baixo teor de fenilalanina, que têm menor custo e maior facilidade de administração ${ }^{14}$. Não se deve retirar totalmente a fenilalanina da dieta, uma vez que a mesma é importante para síntese de proteínas estruturais e funcionais, como a insulina ${ }^{14}$.

Os benefícios do tratamento da PKU são evidentes, tanto entre as crianças para prevenção do comprometimento progressivo das funções cerebrais, como na gestação para se evitarem alterações funcionais e estruturais no desenvolvimento fetal ${ }^{14}$. No entanto, durante a gestação, ganha importância a época de início do tratamento, pois obtêm-se melhores resultados quando o tratamento é iniciado antes da concepção ou durante as primeiras semanas do primeiro trimestre de gestação ${ }^{7,16,19}$. Os efeitos deletérios sobre o feto diminuem quando a fenilalaninemia materna é mantida entre 120 a $400 \mu \mathrm{mol} / \mathrm{L}$ ( 2 a $6 \mathrm{mg} / \mathrm{dL}$ ). Níveis superiores a $400 \mu \mathrm{mol} / \mathrm{L}$ (>6 mg/dL) estão associados a baixo peso ao nascer e leve rebaixamento do $\mathrm{QI}^{19,20}$.

Conforme estes dados, em nossa paciente o início do tratamento não ocorreu no momento ideal, pois ela descobriu ser portadora da doença a partir dos exames realizados no pré-natal, não fazendo controle dietético para PKU previamente à concepção. A gestante foi encaminhada ao prénatal especializado com 13 semanas de gestação, sendo então iniciada a dieta com restrição de fenilalanina, obtendo controle irregular da fenilalanina plasmática devido à adesão deficiente da paciente à dietoterapia. Segundo a literatura, os fatores que podem estar associados para a descontinuidade da dieta são a limitação financeira devido ao elevado custo dos alimentos especiais, desconhecimento dos teores de fenilalanina nos alimentos, falta de tempo para adequar-se à dietoterapia e desconhecimento das implicações dieta-doença ${ }^{14}$. Além disso, do ponto de vista sensorial, estas misturas têm odor e paladar desagradável, dificultando ainda mais a completa adesão das pacientes ${ }^{14}$. Mesmo com níveis variáveis de fenilalanina plasmática durante a gravidez, houve redução importante do valor inicial de fenilalanina $(24 \mathrm{mg} / \mathrm{dL})$ na paciente estudada.

Pode-se afirmar, a partir do resultado perinatal da gestação atual, que houve efeito direto do controle dietoterápico materno, mesmo que irregular, sobre o prognóstico fetal. O RN a termo não apresentou alterações físicas ou defeitos congênitos confirmados. A gestação anterior, na qual não houve diagnóstico e controle da PKU, resultou em criança com séria deficiência psico-motora confirmada, além de microcefalia e distúrbios auditivos e da fala.

Com o conhecimento dos efeitos da hiperfenilalaninemia materna sobre o feto, tornam-se essenciais o diagnóstico e a instituição precoce do tratamento durante a gravidez em pacientes com suspeita clínica de PKU. No caso aqui descrito, houve benefícios materno-fetais do tratamento dietoterápico oferecido, reforçando a importância da identificação de mulheres fenilcetonúricas em idade reprodutiva.

\section{ABSTRACT}

Maternal phenylketonuria is an aminoacid pathology characterized by elevated plasma levels of phenylalanine in the pregnant woman that may cause abnormalities in fetus development, and which is called maternal phenylketonuria syndrome. As the clinical manifestations are non-specific, the disease should be diagnosed by laboratory screening. We present a case of a second pregnancy in a woman with a history of psycho-cognitive development retardation without previous obstetric history, with diagnosis of phenylketonuria in the present gestation, treated with specific phenylalaninefree diet. The newborn did not present congenital defects. The previous gestation without maternal treatment resulted in a child with serious developmental disturbances, microcephalia and auditory-speaking deficits. Early diagnosis and treatment of hyperphenylalaninemia during pregnancy are essential, mainly because of the negative impact on fetal development. In the here reported case, there were fetal benefits from the maternal dietary treatment, which demonstrates the importance of the maternal diagnosis of phenylketonuria in women in reproductive age.

KEYWORDS: Phenylketonuria. Congenital metabolic errors. Hyperphenylalaninemia. High risk pregnancy.

\section{Referências}

1. Longo N. Distúrbios hereditários do metabolismo e do armazenamento de aminoácidos. In: Braunwald E, Fauci AS, Kasper DL, Hauser SL, Longo DL, Jameson JL, editors. Harrison Medicina Interna. 15 ed. Rio de Janeiro: McGraw Hill; 2002. p. 2450-1.

2. Scriver CR, Kaufman S, Woo SCL. The hyperphenylalaninemias. In: Scriver CR, Beaudet AL, Sly WS, Valle D, editors. The metabolic, basis of inherited diseases. $6^{\text {th }}$ ed. New York: McGraw Hill; 1989. p. 495-546. 
3. Jardim LB, Leite JCL, Silveira EL, Barth ML, Giugliani R. Resultados preliminares de um programa de detecção precoce para o hipotireodismo congênito no Rio Grande do Sul. J Pediatr (RJ) 1992; 68:239-42.

4. Koch R, Friedman EG, Wenz E, Jew K, Crowley C, Donnell G. Maternal phenylketonuria. J Inherit Metab Dis 1986; 9 (Suppl 2):159-68.

5. Shils ME, Olson JA, Moshe AS, Ross C. Tratado de nutrição moderna na saúde e na doença. 9 9 $^{\mathbf{a}}$ ed. São Paulo: Manole; 2002.

6. Campistol Plana J, Arellano Pedrola M, Poo Argüelles P, Escofet Sotera C, Perez Olarte P, Vilaseca Busca MA. Embriopatía por fenilcetonuria materna. Una causa de retardo mental poco diagnosticada. Revisión de ocho observaciones. An Esp Pediatr 1999; 51:139-42.

7. Michals-Malaton K, Platt LD, Acosta P, Azen C, Walla CA. Nutrient intake and congenital heart defects in maternal phenylketonuria. Am J Obstet Gynecol $2002 ; 187: 441-4$

8. Platt LD, Koch R, Hanley WB, et al. The international study of pregnancy outcome in women with maternal phenylketonuria: report of a 12-year study. Am J Obstet Gynecol 2000; 182:326-33.

9. Schoonheyt WE, Clarke JT, Hanley WB, Johnson JM, Lehotay DC. Feto-maternal plasma phenylalanine concentration gradient from 19 weeks gestation to term. Clin Chim Acta 1994; 225:165-9.

10.Lenke RR, Levy HL. Maternal phenylketonuria and hyperphenylalaninemia: an international survey of the outcome of untreated and treated pregnancies. N Engl J Med 1980; 303:1202-8.

11.Rouse B, Azen C, Koch R, et al. Maternal Phenylketonuria Collaborative Study (MPKUS) offspring. Facial anomalies, malformations, and early neurological sequelae. Am J Med Genet 1997; 69:89-95.
12.Jardim LB, Palma-Dias R, Silva LC, Ashton-Prolla P, Giuliani R. Maternal hyperphenylalaninaemia as a cause of microcephaly and mental retardation. Acta Paediatr 1996; 85:943-6.

13.Waisbren SE, Levy HL. Effects of untreated maternal hyperphenylalaninemia on the fetus: further study of families identified by routine cord blood screening. J Pediatr 1990; 116:926-9.

14. Mira NVM, Marquez UML. Importância do diagnóstico e tratamento da fenilcetonúria. Rev Saúde Pública 2000; 34:86-96.

15.Levy HL, Lobbregt D, Barnes PD, Poussaint TY. Maternal phenylketonuria: magnetic resonance imaging of the brain in offspring. J Pediatr 1996; 128:770-5.

16.Levy HL, Ghavami M. Maternal phenylketonuria: a metabolic teratogen. Teratology 1996; 53:176-84.

17.Koch R, Blaskovics M. Four cases of hyperphenylalaninaemia: studies during pregnancy and of the offspring produced. J Inherit Metab Dis 1982; 5:11-5.

18.Purnell H. Phenylketonuria and maternal phenylketonuria. Breastfeed Rev 2001; 9:19-21.

19.Levy HL, Waisbren SE, Lobbregt D, et al. Maternal non-phenylketonuric mild hyperphenylalaninemia. Eur J Pediatr 1996; 155 (Suppl 1):S20-5.

20.Whitehead H, Holmes J, Roberts R, et al. Maternal phenylketonuria 1987 to 1993 , pregnancy outcome and early infant development: the Northern Ireland experience. Br J Obstet Gynaecol 1996; 103:1041-4.
Recebido em: 18/5/2004 Aceito com modificações em: 16/9/2004 